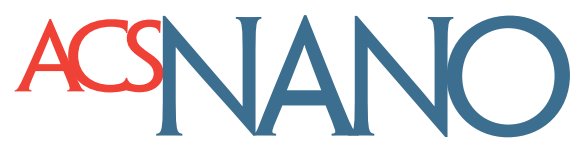

\title{
Gold Chain Formation via Local Lifting of Surface Reconstruction by Hot Electron Injection on $\mathrm{H}_{2}\left(\mathrm{D}_{2}\right) / \mathrm{Au}(111)$
}

\author{
${ }_{4}$ P. Merino,* A. Rosławska, A. Grewal, C. C. Leon, C. Gonzalez, K. Kuhnke, and K. Kern
}

Cite This: https://dx.doi.org/10.1021/acsnano.0c05507

Read Online

\section{ACCESS}

山ll Metrics \& More

回国 Article Recommendations

SI Supporting Information

5 ABSTRACT: The hexagonal close packed surface of gold 6 shows a $22 \times \sqrt{3}$ "herringbone" surface reconstruction which 7 makes it unique among the (111) surfaces of all metals. This 8 long-range energetically favored dislocation pattern appears in 9 response to the strong tensile stress that would be present on 10 the unreconstructed surface. Adsorption of molecular and 11 atomic species can be used to tune this surface stress and lift the 12 herringbone reconstruction. Here we show that herringbone 13 reconstruction can be controllably lifted in ultrahigh vacuum at 14 cryogenic temperatures by precise hot electron injection in the 15 presence of hydrogen molecules. We use the sharp tip of a

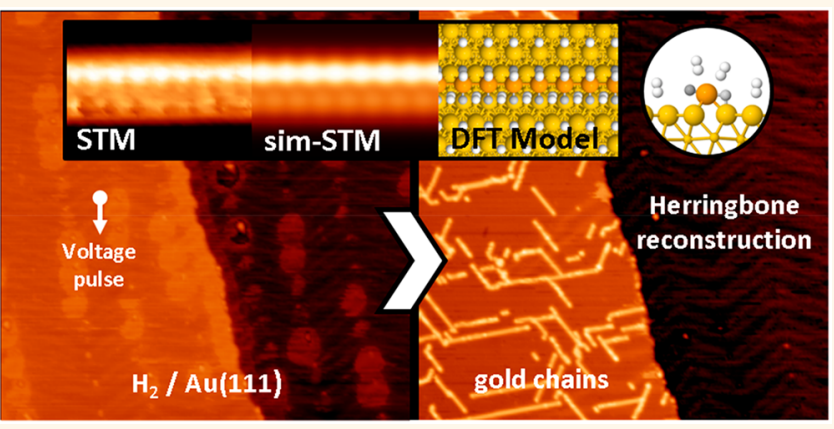
16 scanning tunneling microscope (STM) for charge carrier

17 injection and characterization of the resulting chain nanostructures. By comparing STM images, rotational spectromicroscopy 18 and $a b$ initio calculations, we show that formation of gold atomic chains is associated with release of gold atoms from the 19 surface, lifting of the reconstruction, dissociation of $\mathbf{H}_{2}$ molecules, and formation of surface hydrides. Gold hydrides grow in a 20 zipper-like mechanism forming chains along the [1이 directions of the $\mathrm{Au}(111)$ surface and can be manipulated by further 21 electron injection. Finally, we demonstrate that $\mathrm{Au}(111)$ terraces can be transformed with nearly perfect terrace selectivity 22 over distances of hundreds of nanometers.

23 KEYWORDS: herringbone reconstruction, gold chain, molecular hydrogen, gold hydride, STM

24
25
26 he $22 \times \sqrt{3}$ herringbone reconstruction of $\mathrm{Au}(111)$ consists of a tensile stress-induced periodic arrangement that leads to a dislocation pattern separating 27 regions of fcc and hcp stacking. The stacking domains are 28 separated by soliton walls which form a secondary structure 29 resulting in partial dislocation elbow sites which imprint the 30 characteristic "herringbone" aspect of the surface. ${ }^{1}$ In the top 31 layer, 23 atoms are laterally compressed to fit into a length 32 corresponding to 22 atoms from the bulk along the [011] 33 direction. $^{2}$ By inducing this reconstruction the $\mathrm{Au}(111)$ 34 surface relaxes its intrinsic tensile stress by $22 \% .^{3}$ Other 35 mechanisms for releasing this stress have been found at gold 36 interfaces permitting nanoscale surface engineering. "Magic 37 gold fingers", one atom layer in height, can be formed on the 38 (111) surface of gold via nanomanipulations driven by 39 localized electric field with a scanning tunneling microscope 40 (STM). ${ }^{2,5}$ Relaxations of the herringbone reconstruction are 41 also observed in electrochemistry experiments. ${ }^{6}$ When reactive 42 species such as chlorine ${ }^{7}$ or sulfur ${ }^{8}$ are deposited on $\mathrm{Au}(111)$ 43 in ultrahigh vacuum condition (UHV) they promote the 44 formation of adatom-adsorbate complexes that lift the reconstruction. ${ }^{9,10}$ Alkali metals deposited on $\mathrm{Au}(111)$ also 45 distort the periodicity of the reconstruction by influencing the 46 elastic stress of the topmost layer. ${ }^{11}$ Other adsorbed species 47 such as $\mathrm{O}$ atoms, iron phthalocyanine or perylene, induce 48 partial lifting of the $22 \times \sqrt{3}$ reconstruction due to anisotropic 49 surface stress induced by interfacial charge transfer. ${ }^{12,13} 50$ Hydrogen $\left(\mathrm{H}_{2}\right)$ and its isotope deuterium $\left(\mathrm{D}_{2}\right)$ have been 51 investigated at the atomic-scale adsorbed on $\mathrm{Au}(111)$ but no 52 effect on the herringbone reconstruction has been reported to 53 date. $^{14-16}$ Hydrogen molecules physisorb and develop into 54 coverage dependent superstructures. At low concentrations 55 they form a surface-state mediated Fermi superlattice, ${ }^{17}$ while 56 at coverages close to one monolayer they aggregate into close 57

Received: July 3, 2020

Accepted: October 20, 2020 

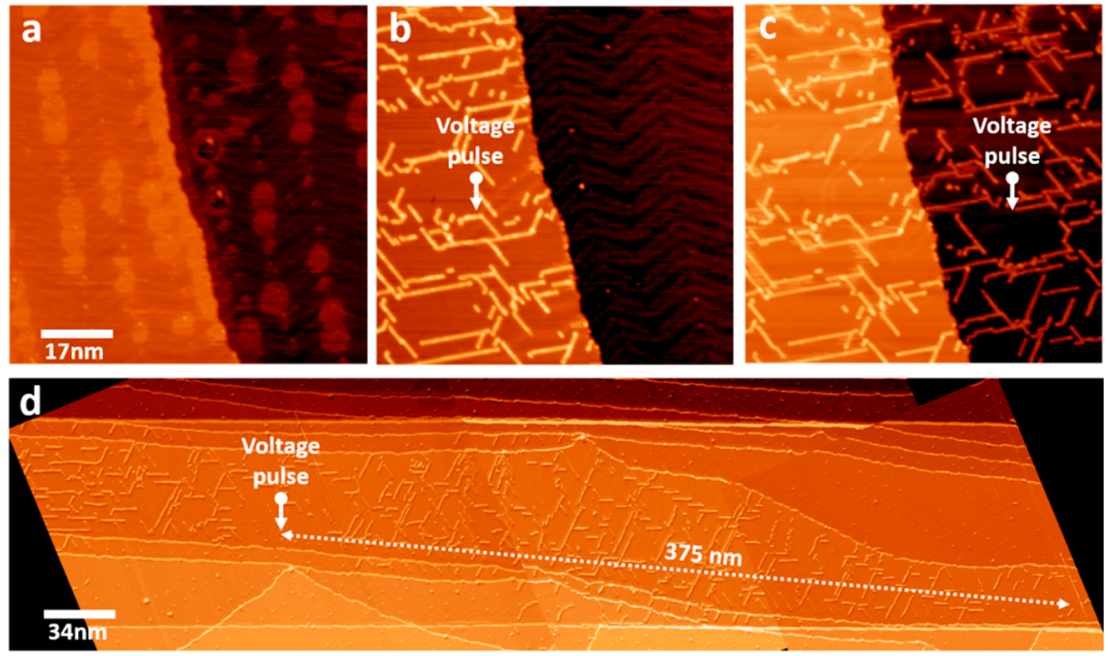

Figure 1. Mesoscopic extension and terrace selectivity of the chain formation. (a)-(c). STM images showing the formation of chains on the $\mathrm{Au}(111)$ surface and lifting of the herringbone reconstruction. Single voltage pulses were applied at the indicated positions, a first pulse on the left-hand side (upper terrace) between images (a) and (b) and a second pulse on the right-hand side (lower terrace) between images (b) and (c). Size: $85 \times 85 \mathrm{~nm}^{2}, 100 \mathrm{pA}$. a) $\left.+20 \mathrm{mV}, \mathrm{b}\right)$ and c) $+2.7 \mathrm{~V}$. (d). The long-range of the chain-creation process is demonstrated by the extended topography merged together from three individual $200 \times 200 \mathrm{~nm}^{2}$ images.

58 packed two-dimensional clusters, ${ }^{18,19}$ in all cases leaving the 59 surface herringbone reconstruction intact.

60 The interaction between hydrogen and gold atoms has been 61 predicted to be strong but dissociating $\mathrm{H}_{2}$ molecules normally 62 needs activation on a catalyzer. ${ }^{20}$ Gold is renowned as the 63 noblest of all metals; ${ }^{21}$ yet, gold nanostructures can show high 64 catalytic activity for many reduction and oxidation reac65 tions, $^{22-24}$ and in particular for dihydrogen dissociation. ${ }^{25-27}$ 66 Important size effects have been observed leading to the 67 conclusion that the edges and corners of the nanostructures are 68 the active sites for dissociation. ${ }^{28,29}$ For this reason, flat gold 69 surfaces are considered to be chemically inert for bond 70 dissociation, as they present a low amount of undercoordinated 71 atoms. $^{30}$ Engineering routes that increase the number of edge 72 or corner atoms per unit area on $\mathrm{Au}(111)$ would boost the 73 catalytic activity of the otherwise inert surface for the study of 74 hydrogenation reactions.

75 Here, we show that the herringbone reconstruction can be 76 locally lifted on individual terraces by applying voltage pulses 77 with the tip of a scanning tunneling microscope (STM) in the 78 presence of physisorbed $\mathrm{H}_{2}$ and $\mathrm{D}_{2}$ molecules. By injecting hot 79 electrons, the " $23 \mathrm{rd}$ extra atom" of each unit cell gets promoted 80 from the top layer onto the terrace and stabilizes the formation 81 of $\mathrm{Au}$ monatomic chains along the $[1 \overline{1} 0]$ directions of the $82 \mathrm{Au}(111)$ surface. We rationalize these experimental findings 83 with extensive ab initio calculations and STM simulations, 84 confirming that upon Au adatom lifting, hydrogen molecules 85 spontaneously dissociate and covalently bond with Au to form 86 interfacial gold hydrides that trigger chain formation in a 87 zipper-like mechanism.

\section{RESULTS AND DISCUSSION}

89 Figure $1 \mathrm{a}-\mathrm{c}$ show a sequence of three STM images illustrating 90 the chain generation process. Two $\mathrm{Au}(111)$ terraces are 91 present on the left and right-hand sides of the images separated 92 by a monatomic step. Figure 1a shows the surface prior to 93 application of voltage pulses. Hydrogen physisorbed on the 94 terraces forms disk-shaped islands decorating the elbows of the 95 herringbone reconstruction (Figure 1a). ${ }^{1,18,19}$ The arrow in
Figure $1 \mathrm{~b}$ marks the position in the left terrace where we 96 applied a $+10 \mathrm{~V}$ voltage pulse for $100 \mathrm{~ms}$ (with positive 97 polarity implying the injection of electrons from the tip to the 98 surface). After the first pulse, the herringbone reconstruction is 99 lifted and one-dimensional nanostructures cover the terrace 100 where the voltage pulse was applied. The structures 101 preferentially align with the three high-symmetry directions, 102 equivalent to the $[1 \overline{1} 0]$ direction of the $\mathrm{Au}(111)$ surface. In a 103 second step, another voltage pulse was applied on the right- 104 hand-side terrace transforming the second, still herringbone 105 reconstructed terrace in the same way as described before (see 106 Figure 1c). The chain generation process is limited to one 107 terrace but extends over large distances. A voltage pulse under 108 tunneling conditions promotes transient injection of hot 109 electrons (i.e., electrons with energies hundreds of times 110 greater than the available thermal energy). ${ }^{31}$ The strict terrace 111 selectivity suggests that the observed transformation process is 112 mediated by the electron surface state, ${ }^{32}$ although other 113 mechanisms involving charge transport on the surface, such as 114 propagation of surface plasmon polaritons, may also play a 115 role.

Inspecting the range of transformation, we find that a single 117 pulse can generate chains more than $350 \mathrm{~nm}$ away from the 118 position where the pulse was applied (Figure 1d). The chains 119 are straight and can be very long $(>20 \mathrm{~nm})$ but encounters 120 with other chains limit their growth. We observe that the chain 121 formation probability saturates at $100 \%$ for pulses of $100 \mathrm{~ms} 122$ duration and $+7 \mathrm{~V}$ bias (see Figure S5 in the Supporting 123 Information (SI)). Chains can also be produced at lower bias 124 voltages but with lower efficiency. Regardless of how chains are 125 made, once they have formed, their shape can be modified with 126 further pulsing (see SI Figure S6). Evaluation of their structure 127 indicates that the number of chain atoms is approximately one 128 adatom per 22 top layer atoms for sufficiently strong pulses, 129 which suggests that the chains consist exclusively of Au atoms 130 expelled from the interface upon lifting the herringbone 131 reconstruction (see SI Figure S1). For milder pulses, both 132 chains and a residual herringbone reconstruction can coexist 133 on the same terrace. 
135 In Figure 2 we present high-resolution images of a short 136 individual chain formed by a voltage pulse on a surface covered
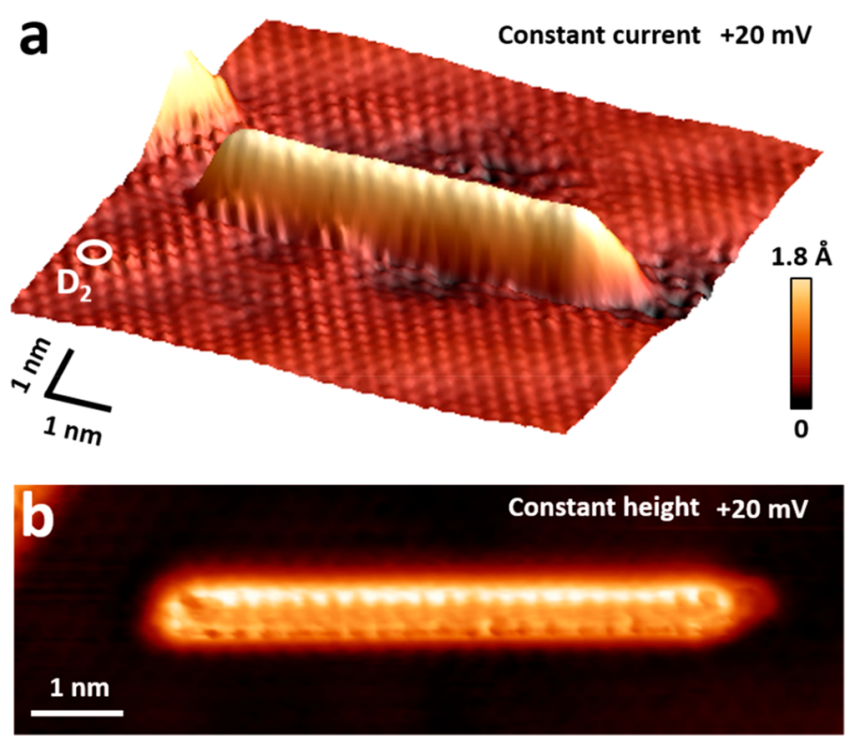

Figure 2. High-resolution images of a short chain. (a) Threedimensional topographic STM image of a characteristic atomic chain. Individual $\mathrm{D}_{2}$ molecules covering the whole area in a dense phase are resolved on the surface (one molecule is marked within a white ellipse) as a hexagonal pattern and on the chain. Nine $\times 9$ $\mathrm{nm}^{2}, 30 \mathrm{pA},+20 \mathrm{mV}$. (b) Constant height current map of a quasi1D chain measured showing atomic resolution. Every protrusion on the chain can be ascribed to a single $D_{2}$ molecule, $9 \times 3 \mathrm{~nm}^{2}$, $+20 \mathrm{mV}$.

137 by isotopic hydrogen molecules (deuterium $\mathrm{D}_{2}$ ) injected into 138 the chamber to replace the residual $\mathrm{H}_{2}$ gas. Figure $2 \mathrm{a}$ shows a 139 pseudo 3D representation of a constant current measurement 140 obtained at a bias voltage of $+20 \mathrm{mV}$. The chain is $6.5 \mathrm{~nm}$ long 141 and has an apparent height of $1.55 \AA$. Individual atomic-scale 142 protrusions are resolved on top of the chain. The features are 143 better resolved in the constant height STM image presented in 144 Figure $2 b$, which reveals that the chain consists of two rows of 145 atomic-scale features where one appears higher than the other. 146 At $+20 \mathrm{mV}$ the molecules exhibit a high density of states and 147 enhanced conductivity characteristic of physisorption. There148 fore, the protrusions can be attributed to individual $\mathrm{D}_{2}$ 149 molecules physisorbed on the chain rather than being 150 chemisorbed to $\mathrm{Au}$ atoms. Indeed, a close packed layer of $151 \mathrm{D}_{2}$ molecules can be resolved as a $2 \mathrm{D}$ mantle covering the 152 terrace (one molecule is marked in Figure 2a).

153 To gain further insight we perform local tunneling 154 spectroscopy on the chains. Figure 3 a shows a $d I / d V$ spectrum 155 of $\mathrm{D}_{2} / \mathrm{Au}(111)$ featuring the surface state of $\mathrm{Au}(111)$ at -0.5 $156 \mathrm{eV}$ and the vibrational peak structure around the Fermi level 157 characteristic for $\mathrm{D}_{2}$ on gold surfaces. ${ }^{33,34}$ The electronic states 158 between $-40 \mathrm{mV}$ and $+40 \mathrm{mV}$ and the two negative 159 differential conductance peaks correspond to the opening of 160 rotational and vibrational channels in inelastic tunneling. The 161 energy and relative intensity of the vibrational peaks is very 162 sensitive to the, isotopic species, the nanocavity size and the 163 exact adsorption configuration of the molecules on the 164 surface. $^{33,34}$ Figure $3 \mathrm{c}$ shows a constant height $\mathrm{d} I / \mathrm{d} V$ map at $165 V_{\text {bias }}=+20 \mathrm{mV}$. The presence of $\mathrm{D}_{2}$ molecules covering the 166 chain can be deduced from the high intensity of the $\mathrm{D}_{2}$-related 167 state measured on top of it. In addition, individual $\mathrm{D}_{2}$
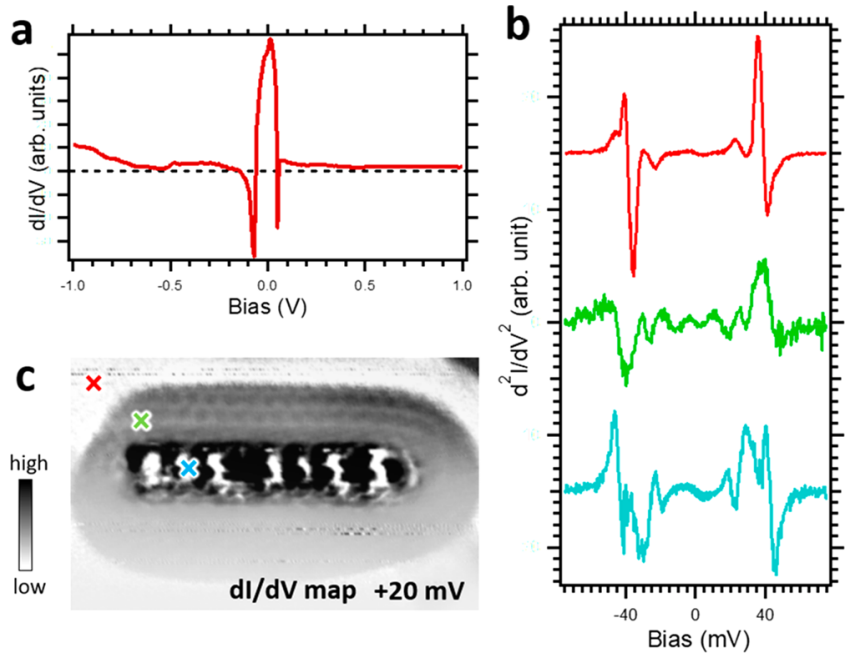

Figure 3. Spectromicroscopy characterization of a short chain generated using $D_{2}$ (a) Wide range $(-1 \mathrm{~V}$ to $+1 \mathrm{~V}) \mathrm{dI} / \mathrm{dV}$ spectrum of the $\mathrm{D}_{2} / \mathrm{Au}(111)$ system. (b) Inelastic tunneling electron spectroscopy as measured by $\mathrm{d}^{2} I / \mathrm{d} V^{2}$ of the $\mathrm{D}_{2} / \mathrm{Au}(111)$ chains. The positions where the individual spectra were measured are marked by color-coded crosses in panel c. The intensity of the red curve was divided by a factor of 4 . (c) Chain mapped under constant height $\mathrm{d} I / \mathrm{d} V$ condition at $+20 \mathrm{mV}$ using a functionalized tip. The chain is surrounded by an oblong island of dense $D_{2}$ molecules, the structure of which can be resolved near the upper edge in the image, size: $6.9 \times 3.8 \mathrm{~nm}^{2}$.

molecules are resolved surrounding the atomic chain forming 168 an oblong island. ${ }^{18,19}$ In Figure $3 \mathrm{~b}$ we present $\mathrm{d}^{2} I / \mathrm{d} V^{2}$ spectra 169 obtained on the positions marked in Figure 3c. Strikingly, the 170 $\mathrm{d}^{2} I / \mathrm{d} V^{2}$ spectrum obtained on the region free of $\mathrm{D}_{2}$ molecules 171 (red color) shows sharp peaks at $\pm 40 \mathrm{meV}$ with $5 \mathrm{meV}$ width 172 which is a strong indication of tip functionalization with a 173 picked-up molecule. Functionalization enhances contrast and 174 lateral resolution. ${ }^{14,15,35-37}$ In addition, the molecule at the tip 175 increases the sensitivity to the local environment and the 176 spatial variations of the potential energy of the surface which 177 permits one to perform, so-called, rotational spectro-micros- 178 copy maps. ${ }^{16}$ Accordingly, Figure $3 c$ charts the interaction 179 between deuterium on the tip and on the sample. The $d^{2} I / d V^{2} 180$ spectrum recorded on the dense $\mathrm{D}_{2}$ island (green curve) shows 181 broader $(10 \mathrm{meV})$ peaks and twice the number of features, 182 indicative of vibrational coupling between the molecule at the 183 tip and those on the surface. ${ }^{38,39}$ The $\mathrm{d}^{2} I / \mathrm{d} V^{2}$ spectrum 184 recorded on top of the chain (blue curve) also exhibits twice as 185 many peaks and dip features, some of them being broad (10 186 $\mathrm{meV})$ and some being sharp $(5 \mathrm{meV})$. These observations 187 indicate that $\mathrm{D}_{2}$ molecules on top of the chain interact less 188 strongly with $\mathrm{D}_{2}$ at the tip than the molecules in the densely 189 packed island surrounding the chain.

190

In Figure $4 \mathrm{a}$ we present the ball-and-stick model of the $191 \mathrm{f} 4$ energetically most stable structure determined by our density 192 functional theory (DFT) simulations. It consists of a 193 concatenation of gold adatoms covalently bonded with two 194 hydrogen atoms at the sides of the chain. One of the two 195 hydrogen atoms bonds exclusively to the adatom (depicted in 196 dark gray in Figure 4a) and the other bridges between the 197 adatom and a Au atom on the surface (depicted in light gray). 198 The whole structure is fully covered with a monolayer of 199 vertically aligned physisorbed $\mathrm{H}_{2}$ molecules responsible for the 200 atomic-scale features resolved in the STM images and $\mathrm{d} I / \mathrm{d} V 201$ 

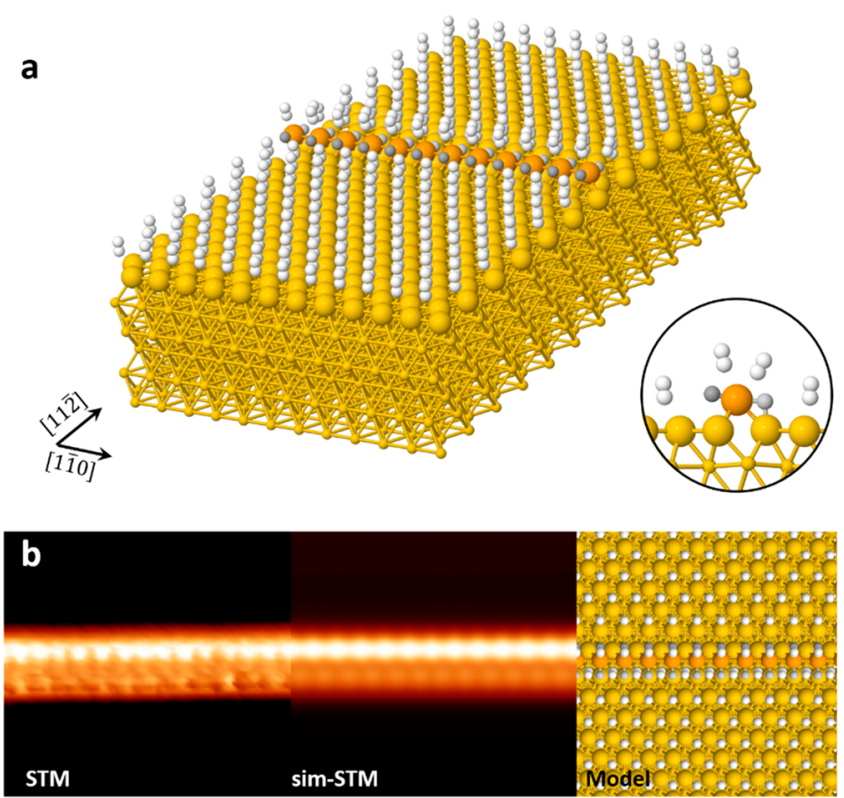

C

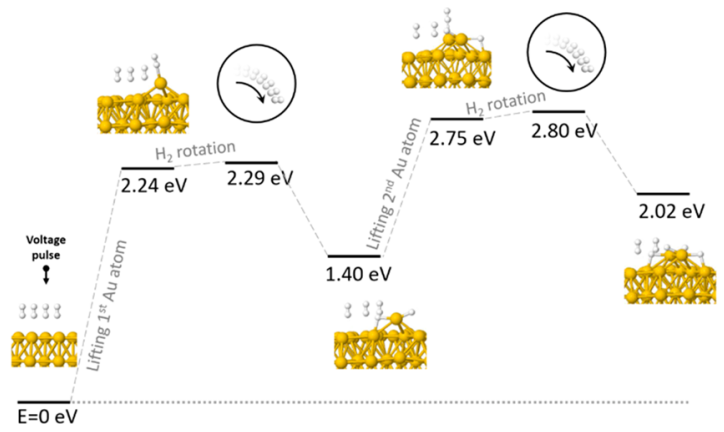

Figure 4. Theoretical structural model, STM simulation and mechanism for chain formation. (a) Ball-and-stick model of the lowest energy structure. Yellow and white balls correspond to gold and hydrogen atoms, respectively. The orange balls represent the $\mathrm{Au}$ atoms forming chains aligned with the[10] directions of the $\mathrm{Au}(111)$ surface. Light and dark gray balls represent $H$ atoms originating from a dissociated molecule. Inset: Side view of the ball-and-stick model of the best fit structure. The vertically aligned hydrogen molecules cover the surface and the hydrogen atoms are asymmetrically bound to the Au chain. (b) Constant height STM image (left-hand side) of an atomic chain, constant height STM simulation (middle), and top view of the ball-and-stick structure (right-hand side). The maxima in the simulation correspond to the positions of the hydrogen molecules physisorbed on the chain. (c) Schematic representation of the initial steps involved in the chain formation from DFT calculations: Au-lifting, $\mathrm{H}_{2}$ rotation, Auinduced hydrogen dissociation, next-neighbor Au lifting. The diagram associated with the lowest barrier reaction pathway reveals a mechanism involving the energy barriers that have to be overcome by the energy provided by the injected hot electrons.

202 maps. In Figure $4 \mathrm{~b}$ we present a comparison between the STM 203 simulation of the structure and the experimental images. On 204 the left-hand side is the measurement, in the middle the STM 205 simulation, and on the right-hand side the top view of the ball206 and-stick model. The agreement between experimental and 207 simulated STM images is very good. The $\mathrm{H}_{2}$ molecules 208 physisorbed over the chain form the characteristic zigzag 209 pattern observed in the experimental STM image. The two 210 rows of $\mathrm{H}_{2}$ molecules are physisorbed at different heights over 211 the chains, due to the asymmetric $\mathrm{Au}-\mathrm{H}$ bonding config- uration. The height difference is resolved in the STM image 212 simulation, as one of the rows appears brighter than the other 213 thus reproducing the small asymmetry observed in the chain 214 topography.

215

$A b$ initio calculations permit us to determine the formation 216 mechanism of the atomic chains. We start our analysis by 217 reproducing the herringbone reconstruction building a slab 218 with the $(22 \times \sqrt{3})$ periodicity including six layers of Au 219 atoms in the unit cell (see the Supporting Information for 220 details)..$^{40}$ In our calculations, when the "extra 23rd atom" of 221 the herringbone reconstruction is placed as an adatom on a 222 pristine $\mathrm{Au}(111)$ unreconstructed surface, it returns back to 223 the top bulk layer upon relaxation of the system confirming 224 that theory reproduces the fact that the herringbone 225 reconstruction is energetically favorable.

226

We have simulated and evaluated the chain formation by 227 dividing the process in steps. In Figure 4c we present a scheme 228 showing the energy of the initial intermediate states. In a first 229 step, we start pulling up one of two "extra $23^{\text {rd }}$ atoms" from the 230 $22 \times \sqrt{3}$ unit cell with an energy cost of $2.24 \mathrm{eV}$. The 231 calculations have been made with a doubled $22 \times \sqrt{3}$ unit cell, 232 that is, $22 \times 2 \sqrt{3}$, which leaves the other three "extra 23rd 233 atoms" embedded in the surface. The following step considers 234 the rotation of one $\mathrm{H}_{2}$ molecule from its original orientation 235 (perpendicular to the surface) to a parallel position on the 236 lifted atom. This process increases the energy of the system by 237 $0.05 \mathrm{eV}$. When a hydrogen molecule is physisorbed parallel to 238 the surface on top of the gold adatom, the highly under- 239 coordinated gold adatom catalyzes its exothermic dissociation 240 forming a stable interfacial Au-hydride chemisorbed complex. 241 The system further relaxes by migrating one of the hydrogen 242 atoms in a bridge position between the adatom and the nearest 243 neighboring $\mathrm{Au}$ atom located in the surface. The total energy 244 gain by the system upon $\mathrm{H}_{2}$ dissociation and hydride formation 245 is $-0.89 \mathrm{eV}$.

246

In a second step, another $\mathrm{Au}$ atom is promoted to the 247 interface. It will preferably be the next atom in [1 $\overline{1} 0]$ direction 248 neighboring the first formed hydride (see SI Figure S9 in the 249 Supporting Information). This newly expelled atom is released 250 from the next row of compressed atoms in the surface 251 reconstruction. The total energy cost of this process is $1.35 \mathrm{eV} .252$ Since these $1.35 \mathrm{eV}$ for pulling out a second gold adatom are 253 $0.89 \mathrm{eV}$ lower than the $2.24 \mathrm{eV}$ required to lift the first atom, 254 the system will tend to form atomic chains in a coordinated 255 zipper-like fashion rather than expelling atoms at separated 256 locations. After rotating the $\mathrm{H}_{2}$ on top, the second adatom 257 spontaneously dissociates the molecule and forms a second 258 gold hydride complex with an energy gain of $-0.78 \mathrm{eV}$. We 259 speculate that the hot electrons injected by the voltage pulse 260 excite out-of-plane phonon modes that help to pull $\mathrm{Au}$ atoms 261 above the surface plane. The dissociation of the very first $\mathrm{H}_{2} 262$ molecule by the undercoordinated $\mathrm{Au}$ adatom results in 263 nucleating a chain that leads to continued lifting of 264 neighboring atoms in a zipper-like fashion. This process in 265 turn leads to quasi-1D chains observed in the experiment. 266 Surface diffusion simulations further corroborate the zipper- 267 like chain growth mechanism, as the formation of $2 \mathrm{D}$ clusters 268 would be favored over nm-long linear chains when we assume 269 nucleation from randomly generated gold hydride monomer 270 and on-surface diffusion (see Supporting Information). 271

The final $22 \times 2 \sqrt{3}$ structure with all four Au "extra $23^{\text {rd }} 272$ atoms" following the $[1 \overline{1} 0]$ direction promoted as adatoms, the 273 
274 four $\mathrm{H}_{2}$ molecules dissociated, the herringbone reconstruction 275 lifted and the development of a $(1 \times 1) \mathrm{Au}(111)$ surface has a 276 total energy $3.76 \mathrm{eV}$ higher than the initial structure. Given the 277 large energy barriers involved in the adatom lifting process, we 278 propose that the injection of hot electrons helps the system 279 surmount these barriers and reach a local energy minimum 280 which is stabilized at the cryogenic temperatures of the 281 experiment. Indeed, gold nanowires are known to dissociate 282 hydrogen molecules with very low activation barriers. ${ }^{20,29,41,42}$ 283 Note that in order to simplify the calculations we have used 284 only four $\mathrm{H}_{2}$ adsorbate molecules in the simulation of the 285 growth process. We estimate that the effect of the full 286 hydrogen monolayer mantle covering the surface is negligible 287 in our analysis of the energetics of the system.

\section{CONCLUSIONS}

289 To conclude, we present a combined experimental and 290 theoretical atomic-scale study that demonstrates the controlled 291 generation of atomic chains on the $\mathrm{Au}(111)$ surface upon hot 292 electron injection with voltage pulses. The mechanism involves 293 the expulsion of the " $23^{\text {rd }}$ extra atom" of the herringbone 294 reconstruction to the surface followed by stabilization in the 295 presence of hydrogen (deuterium) molecules. This process lifts 296 the herringbone reconstruction and forms one-dimensional 297 chains in a terrace selective manner over mesoscopic distances. 298 Combining experiments with $a b$ initio calculations we 299 determine that the chains are aligned with the three equivalent $300[1 \overline{1} 0]$ directions and consist of $\mathrm{Au}$ adatoms stabilized by $\mathrm{H}$ 301 atoms. Hydrogen molecules spontaneously dissociate in the 302 presence of undercoordinated $\mathrm{Au}$ atoms and form chemical 303 bonds with them. The resulting gold hydrides remain stabilized 304 on the surface and further ease the promotion of a first305 neighbor $\mathrm{Au}$ atom to the interface, fostering the formation of 306 the chains in a zipper-like fashion. Finally, the hydride adatom 307 chains are covered with the excess molecules which form a 308 mantle of vertically aligned $\mathrm{H}_{2}\left(\mathrm{D}_{2}\right)$ and determine the atomic 309 scale periodic structure observed in the STM images. Our 310 finding enables locally functionalizing $\mathrm{Au}(111)$ surfaces that 311 may enhance their catalytic activity for hydrogen dissociation 312 and permit the study of hydrogenation chemical reactions at 313 the atomic-scale in a model system.

\section{METHODS/EXPERIMENTAL}

315 Sample Preparation. $\mathrm{Au}(111)$ single crystal samples were 316 cleaned by repeated cycles of $\mathrm{Ar}^{+}$-sputtering and annealing which 317 result in a clean surface with terraces of $10-100 \mathrm{~nm}$ width showing 318 the herringbone reconstruction everywhere on the surface as 319 confirmed with STM. Clean $\mathrm{Au}(111)$ at $\sim 10 \mathrm{~K}$ is exposed to a 320 partial pressure of $\mathrm{D}_{2}$ of $1.5 \times 10^{-7} \mathrm{mbar}$ (Linde Minican $99 \%$ purity) 321 for $2 \mathrm{~h}$ with no line of sight between the leak valve and the sample. 322 Due to this fact and the geometry of the vacuum chamber the 323 adsorbates start to appear on the surface only after several hours of 324 dosing $\mathrm{D}_{2}{ }^{15}$ Alternatively, $\mathrm{H}_{2}$ from residual background gas $\left(\sim 10^{-11}\right.$ 325 partial pressure), as confirmed by a detailed residual gas analysis, 326 present in the UHV cryostat is condensed on the $\mathrm{Au}(111)$ surface and 327 used in the experiments.

STM Measurements. The experiments were conducted in a 329 home-built low-temperature (4.2 K) STM, under ultrahigh vacuum 330 conditions. The $\mathrm{d} I / \mathrm{d} V$ spectra were obtained using a lock-in amplifier, 331 with a modulation voltage of $V_{\mathrm{rms}}=10 \mathrm{mV}$ at $938.5 \mathrm{~Hz}$. Analysis of 332 STM and STS data were performed with the software WSxM. ${ }^{43} \mathrm{~d}^{2} I$ / $333 \mathrm{~d} V^{2}$ spectra were measured by analyzing the second harmonic of the 334 excitation frequency.

335 Theoretical Details. DFT calculations have been performed using 336 the FIREBALL package (see the Supporting Information for details). ${ }^{44}$ We create a herringbone unit cell with a $22 \times \sqrt{3} 337$ periodicity including six slabs of $\mathrm{Au}$ atoms (266 gold atoms). This 338 unit cell has been doubled for the energetic calculations (532 Au 339 atoms plus four $\mathrm{H}_{2}$ molecules). Due to the large lattice vectors, the 340 Brillouin zone (BZ) has been sampled only with the gamma point. 341 The extracted atoms were moved up in steps of $0.25 \AA$ until the 342 required final height is found. In such processes, the Z-coordinate of 343 the uppermost $\mathrm{Au}$ atoms remains fixed. Using this geometry as a 344 starting point, the $\mathrm{H}_{2}$ molecule is rotated and finally it spontaneously 345 dissociates. Following the preferential directions observed in the 346 experiment, the geometry of the final structure should in fact be 347 simulated by using a slightly different $22 \times 2 \sqrt{3}$ periodicity with the 348 exact same size and number of atoms. In the search of the most stable 349 structure, we have tried 16 distinct initial atomic configurations. In a 350 final step, we fully cover the Au surface and the $\mathrm{Au}-\mathrm{H}$ chain with $\mathrm{H}_{2} 351$ molecules, resulting in a structure of 716 atoms. For the STM 352 simulations, we used a homemade package based on the Keldysh- 353 Green function formalism. ${ }^{45}$

354

\section{ASSOCIATED CONTENT}

The Supporting Information is available free of charge at 357 https://pubs.acs.org/doi/10.1021/acsnano.0c05507. 358

$\mathrm{Au}$ chain adatom density; Chain length statistics; Chain 359 morphology; Diffusion simulations; Chain formation 360 efficiency as a function of voltage and current of the 361 pulses; Modification of the chains with consecutive 362 voltage pulses; Supplementary theory and Supplemen- 363 tary references (PDF)

\section{AUTHOR INFORMATION}

\section{Corresponding Author}

P. Merino - Max Planck Institute for Solid State Research, 367 D70569 Stuttgart, Germany; Instituto de Ciencia de Materiales 368 de Madrid, CSIC, E28049 Madrid, Spain; Instituto de Fisica 369 Fundamental, CSIC, E28006 Madrid, Spain; 이이.org/ 370 0000-0002-0267-4020; Email: pablo.merino@csic.es

$$
371
$$

\section{Authors}

A. Rosławska - Max Planck Institute for Solid State Research, 373 D70569 Stuttgart, Germany; (1) orcid.org/0000-0002-0317- 374 1775

A. Grewal - Max Planck Institute for Solid State Research, 376 D70569 Stuttgart, Germany

C. C. Leon - Max Planck Institute for Solid State Research, 378 D70569 Stuttgart, Germany; 이이.org/0000-0003-4132- 379 4645

C. Gonzalez - Departamento de Fisica Téorica de la Materia 381 Condensada and Condensed Matter Physics Center (IFIMAC), 382 Facultad de Ciencias, Universidad Autonoma de Madrid, 383 E28049 Madrid, Spain; Departamento de Fisica de Materiales, 384 Universidad Complutense de Madrid, 28040 Madrid, Spain; 385 Instituto de Magnetismo Aplicado UCM-ADIF, E-28232 Las 386 Rozas de Madrid, Spain

K. Kuhnke - Max Planck Institute for Solid State Research, 388 D70569 Stuttgart, Germany; @ orcid.org/0000-0001-9981- 389 1732

K. Kern - Max Planck Institute for Solid State Research, D70569 Stuttgart, Germany; Institut de Physique, Ecole Polytechnique Féderale de Lausanne, 1015 Lausanne, Switzerland

Complete contact information is available at: https://pubs.acs.org/10.1021/acsnano.0c05507

(1)




\section{Notes}

398 The authors declare no competing financial interest.

\section{ACKNOWLEDGMENTS}

400 C.G. was funded by Spanish Ministry of Science, Innovation 401 and Universities under the projects MAT2017-88258-R and 402 MDM-2014-0377 (Maria de Maeztu Programme for Units of 403 Excellence in R\&D). C.G. acknowledge the computer 404 resources at Cibeles and the technical support provided by 405 the Scientific Computing Center at UAM, project FI-2019406 0028. PM acknowledges support from the A.v. Humboldt 407 Foundation, the ERC Synergy Program (grant no. ERC-2013408 SYG-610256, Nanocosmos), Spanish MINECO (MAT2017409 85089-C2-1-R) and the "Comunidad de Madrid" for its 410 support to the FotoArt-CM Project S2018/NMT-4367 411 through the Program of R\&D activities between research 412 groups in Technologies 2013, cofinanced by European 413 Structural Funds.

\section{REFERENCES}

415 (1) Barth, J. V.; Brune, H.; Ertl, G.; Behm, R. J. Scanning Tunneling 416 Microscopy Observations on the Reconstructed $\mathrm{Au}(111)$ Surface: 417 Atomic Structure, Long-Range Superstructure, Rotational Domains, 418 and Surface Defects. Phys. Rev. B: Condens. Matter Mater. Phys. 1990, 419 42, 9307-9318.

420 (2) Guo, Q.; Yin, F.; Palmer, R. E. Beyond the Herringbone 421 Reconstruction: Magic Gold Fingers. Small 2005, 1, 76-79.

422 (3) Bach, C. E.; Giesen, M.; Ibach, H.; Einstein, T. L. Stress Relief in 423 Reconstruction. Phys. Rev. Lett. 1997, 78, 4225-4228.

424 (4) Hasegawa, Y.; Avouris, P. Manipulation of the Reconstruction of 425 the $\mathrm{Au}(111)$ Surface with the STM. Science 1992, 258, 1763-1765. 426 (5) Yin, F.; Palmer, R.; Guo, Q. Nanoscale Surface Recrystallization 427 Driven by Localized Electric Field. Phys. Rev. B: Condens. Matter 428 Mater. Phys. 2006, 73, 73405.

429 (6) Wu, Q.; Shang, W. H.; Yan, J. W.; Xie, Z. X.; Mao, B. W. Metal 430 Adlayer-Induced Relaxation of $\mathrm{Au}(111)$ Reconstruction under 431 Electrochemical Control. J. Phys. Chem. B 2003, 107, 4065-4069.

432 (7) Andryushechkin, B. V.; Cherkez, V. V.; Gladchenko, E. V.; 433 Pavlova, T. V.; Zhidomirov, G. M.; Kierren, B.; Didiot, C.; Fagot434 Revurat, Y.; Malterre, D.; Eltsov, K. N. Self-Organization of Gold 435 Chloride Molecules on Au(111) Surface. J. Phys. Chem. C 2013, 117, 43624948.

437 (8) Maksymovych, P.; Sorescu, D. C.; Yates, J. T. Gold-Adatom438 Mediated Bonding in Self-Assembled Short-Chain Alkanethiolate 439 Species on the $\mathrm{Au}(111)$ Surface. Phys. Rev. Lett. 2006, 97, 146103. 440 (9) Maksymovych, P.; Voznyy, O.; Dougherty, D. B.; Sorescu, D. C.; 441 Yates, J. T. Gold Adatom as a Key Structural Component in Self442 Assembled Monolayers of Organosulfur Molecules on $\mathrm{Au}(111)$. Prog. 443 Surf. Sci. 2010, 85, 206-240.

444 (10) Cossaro, A.; Mazzarello, R.; Rousseau, R.; Casalis, L.; Verdini, 445 A.; Kohlmeyer, A.; Floreano, L.; Scandolo, S.; Morgante, A.; Klein, M. 446 L.; Scoles, G. X-Ray Diffraction and Computation Yield the Structure 447 of Alkanethiols on Gold(111). Science 2008, 321, 943-946.

448 (11) Barth, J. V.; Behm, R. J.; Ertl, G. Mesoscopic Structural 449 Transformations of the $\mathrm{Au}(111)$ Surface Induced by Alkali Metal 450 Adsorption. Surf. Sci. 1994, 302, L319-L324.

451 (12) Sun, J. T.; Gao, L.; He, X. B.; Cheng, Z. H.; Deng, Z. T.; Lin, 452 X.; Hu, H.; Du, S. X.; Liu, F.; Gao, H.-J. Surface Reconstruction 453 Transition of Metals Induced by Molecular Adsorption. Phys. Rev. B: 454 Condens. Matter Mater. Phys. 2011, 83, 115419.

455 (13) Min, B. K.; Deng, X.; Pinnaduwage, D.; Schalek, R.; Friend, C. $456 \mathrm{M}$. Oxygen-Induced Restructuring with Release of Gold Atoms from $457 \mathrm{Au}(111)$. Phys. Rev. B: Condens. Matter Mater. Phys. 2005, 72,. 458 DOI: $10.1103 /$ PhysRevB.72.121410

459 (14) Weiss, C.; Wagner, C.; Temirov, R.; Tautz, F. S. Direct Imaging 460 of Intermolecular Bonds in Scanning Tunneling Microscopy. J. Am. 461 Chem. Soc. 2010, 132, 11864-11865.
(15) Tautz, R. T.; S. S; O. N; A. C. L; F. S. A Novel Method 462 Achieving Ultra-High Geometrical Resolution in Scanning Tunnelling 463 Microscopy. New J. Phys. 2008, 10, 53012.

(16) Li, S.; Yuan, D.; Yu, A.; Czap, G.; Wu, R.; Ho, W. Rotational 465 Spectromicroscopy: Imaging the Orbital Interaction between 466 Molecular Hydrogen and an Adsorbed Molecule. Phys. Rev. Lett. 467 2015, 114, 206101.

(17) Merino, P.; Rosławska, A.; Leon, C. C.; Grewal, A.; Große, C.; 469 González, C.; Kuhnke, K.; Kern, K. A Single Hydrogen Molecule as an 470 Intensity Chopper in an Electrically Driven Plasmonic Nanocavity. 471 Nano Lett. 2019, 19, 235-241.

(18) Therrien, A. J.; Pronschinske, A.; Murphy, C. J.; Lewis, E. A.; 473 Liriano, M. L.; Marcinkowski, M. D.; Sykes, E. C. H. Collective 474 Effects in Physisorbed Molecular Hydrogen on Ni/Au(111). Phys. 475 Rev. B: Condens. Matter Mater. Phys. 2015, 92, 161407.

(19) Yang, K.; Xiao, W.; Liu, L.; Fei, X.; Chen, H.; Du, S.; Gao, H.-J. 477 Construction of Two-Dimensional Hydrogen Clusters on $\mathrm{Au}(111) 478$ Directed by Phthalocyanine Molecules. Nano Res. 2014, 7, 79-84. 479

(20) Corma, A.; Boronat, M.; Gonzalez, S.; Illas, F. On the 480 Activation of Molecular Hydrogen by Gold: A Theoretical 481 Approximation to the Nature of Potential Active Sites. Chem. 482 Commun. 2007, 3371-3373.

(21) Hammer, B.; Norskov, J. K. Why Gold Is the Noblest of All the 484 Metals. Nature 1995, 376, 238-240.

(22) Zhang, Y.; Cui, X.; Shi, F.; Deng, Y. Nano-Gold Catalysis in 486 Fine Chemical Synthesis. Chem. Rev. 2012, 112, 2467-2505.

(23) Takei, T.; Akita, T.; Nakamura, I.; Fujitani, T.; Okumura, M.; 488 Okazaki, K.; Huang, J.; Ishida, T.; Haruta, M. Heterogeneous 489 Catalysis by Gold. Adv. Catal. 2012, 51, 1-126.

(24) Jaramillo, T. F.; Baeck, S.-H.; Cuenya, B. R.; McFarland, E. W. 491 Catalytic Activity of Supported Au Nanoparticles Deposited from 492 Block Copolymer Micelles. J. Am. Chem. Soc. 2003, 125, 7148-7149. 493

(25) Fujitani, T.; Nakamura, I.; Akita, T.; Okumura, M.; Haruta, M. 494 Hydrogen Dissociation by Gold Clusters. Angew. Chem., Int. Ed. 2009, 495 48 (50), 9515-9518.

(26) Kartusch, C.; van Bokhoven, J. A. Hydrogenation over Gold 497 Catalysts: The Interaction of Gold with Hydrogen. Gold Bull. 2009, 498 42, 343-348.

(27) Mukherjee, S.; Libisch, F.; Large, N.; Neumann, O.; Brown, L. 500 V.; Cheng, J.; Lassiter, J. B.; Carter, E. A.; Nordlander, P.; Halas, N. J. 501 Hot Electrons Do the Impossible: Plasmon-Induced Dissociation of 502 H2 on Au. Nano Lett. 2013, 13, 240-247.

503

(28) Haruta, M. Spiers Memorial Lecture Role of Perimeter 504 Interfaces in Catalysis by Gold Nanoparticles. Faraday Discuss. 505 2011, 152, 11-32. 506

(29) Jelínek, P.; Pérez, R.; Ortega, J.; Flores, F. Hydrogen 507 Dissociation over $\mathrm{Au}$ Nanowires and the Fractional Conductance 508 Quantum. Phys. Rev. Lett. 2006, 96, 46803.

(30) Zanchet, A.; Dorta-Urra, A.; Roncero, O.; Flores, F.; Tablero, 510 C.; Paniagua, M.; Aguado, A. Mechanism of Molecular Hydrogen 511 Dissociation on Gold Chains and Clusters as Model Prototypes of 512 Nanostructures. Phys. Chem. Chem. Phys. 2009, 11, 10122-10131. 513

(31) Lock, D.; Rusimova, K. R.; Pan, T. L.; Palmer, R. E.; Sloan, P. 514 A. Atomically Resolved Real-Space Imaging of Hot Electron 515 Dynamics. Nat. Commun. 2015, 6, 8365.

(32) Schendel, V.; Borca, B.; Pentegov, I.; Michnowicz, T.; Kraft, U.; 517 Klauk, H.; Wahl, P.; Schlickum, U.; Kern, K. Remotely Controlled 518 Isomer Selective Molecular Switching. Nano Lett. 2016, 16, 93-97. 519

(33) Li, S.; Yu, A.; Toledo, F.; Han, Z.; Wang, H.; He, H. Y.; Wu, R.; 520 Ho, W. Rotational and Vibrational Excitations of a Hydrogen 521 Molecule Trapped within a Nanocavity of Tunable Dimension. 522 Phys. Rev. Lett. 2013, 111, 146102.

(34) Wang, H.; Li, S.; He, H.; Yu, A.; Toledo, F.; Han, Z.; Ho, W.; 524 $\mathrm{Wu}, \mathrm{R}$. Trapping and Characterization of a Single Hydrogen Molecule 525 in a Continuously Tunable Nanocavity. J. Phys. Chem. Lett. 2015, 6, 526 $3453-3457$.

(35) Martínez, J. I.; Abad, E.; González, C.; Flores, F.; Ortega, J. 528 Improvement of Scanning Tunneling Microscopy Resolution with H- 529 Sensitized Tips. Phys. Rev. Lett. 2012, 108, 246102. 
531 (36) Chiang, C.; Xu, C.; Han, Z.; Ho, W. Real-Space Imaging of 532 Molecular Structure and Chemical Bonding by Single-Molecule 533 Inelastic Tunneling Probe. Science 2014, 344, 885-888.

534 (37) de la Torre, B.; Švec, M.; Foti, G.; Krejčí, O.; Hapala, P.; 535 Garcia-Lekue, A.; Frederiksen, T.; Zbořil, R.; Arnau, A.; Vázquez, H.; 536 Jelinek, P. Submolecular Resolution by Variation of the Inelastic 537 Electron Tunneling Spectroscopy Amplitude and Its Relation to the 538 AFM/STM Signal. Phys. Rev. Lett. 2017, 119, 166001.

539 (38) Han, Z.; Czap, G.; Xu, C.; Chiang, C.; Yuan, D.; Wu, R.; Ho, $540 \mathrm{~W}$. Probing Intermolecular Coupled Vibrations between Two 541 Molecules. Phys. Rev. Lett. 2017, 118, 36801.

542 (39) Czap, G.; Han, Z.; Wagner, P. J.; Ho, W. Detection and 543 Characterization of Anharmonic Overtone Vibrations of Single 544 Molecules on a Metal Surface. Phys. Rev. Lett. 2019, 122, 106801.

545 (40) Hanke, F.; Björk, J. Structure and Local Reactivity of the $546 \mathrm{Au}(111)$ Surface Reconstruction. Phys. Rev. B: Condens. Matter Mater. 547 Phys. 2013, 87, 235422.

548 (41) Zanchet, A.; Roncero, O.; Dorta-Urra, A.; Aguado, A.; 549 Martínez, J. I.; Flores, F.; Lorente, N. Electron Transport Signature 550 of H2 Dissociation on Atomic Gold Wires. Phys. Rev. B: Condens. 551 Matter Mater. Phys. 2014, 90, 41404.

552 (42) Smit, R. H. M.; Noat, Y.; Untiedt, C.; Lang, N. D.; van Hemert, 553 M. C.; van Ruitenbeek, J. M. Measurement of the Conductance of a 554 Hydrogen Molecule. Nature 2002, 419, 906-909.

555 (43) Horcas, I.; Fernandez, R.; Gomez-Rodriguez, J. M.; Colchero, 556 J.; Gomez-Herrero, J.; Baro, A. M. WSXM: A Software for Scanning 557 Probe Microscopy and a Tool for Nanotechnology. Rev. Sci. Instrum. 558 2007, 78, 13705.

559 (44) Lewis, J. P.; Jelínek, P.; Ortega, J.; Demkov, A. A.; Trabada, D. 560 G.; Haycock, B.; Wang, H.; Adams, G.; Tomfohr, J. K.; Abad, E.; 561 Wang, H.; Drabold, D. A. Advances and Applications in the 562 FIREBALL $a b$ Initio Tight-Binding Molecular-Dynamics Formalism. 563 Phys. Status Solidi B 2011, 248, 1989-2007.

564 (45) González, C.; Abad, E.; Dappe, Y. J.; Cuevas, J. C. Theoretical 565 Study of Carbon-Based Tips for Scanning Tunnelling Microscopy. 566 Nanotechnology 2016, 27, 105201. 